\title{
A RESISTANCE DETERMINANT TO NUCLEIC ACID-BINDING COMPOUNDS IN METHICILLIN-RESISTANT STAPHYLOCOCCUS AUREUS
}

\author{
K. R. Emslie, D. E. Townsend and W. B. GrubB \\ School of Medical Technology, Western Australian Institute of Technology, Kent Street, \\ Bentley, Western Australia, 6102 Australia
}

\begin{abstract}
Summary. Recent isolates of methicillin-resistant Staphylococcus aureus from Australia and several other countries carry a plasmid coding for high levels of resistance to propamidine isethionate and low levels of resistance to cetyltrimethyl-ammonium bromide. The reasons for the acquisition and retention of such a determinant are not known. To define the properties of this resistance determinant in more detail, the minimum inhibitory concentrations of a series of cationic agents, including ethidium bromide, were determined and a cationic-resistance profile prepared for several strains of $S$. aureus and their isogenic, sensitive derivatives. The compounds for which resistance is coded by this determinant all bind to nucleic acids. Hence the determinant has been designated the NAB (nucleic acid-binding compounds)-resistance determinant.
\end{abstract}

\section{INTRODUCTION}

Our laboratory has been studying methicillin-resistant Staphylococcus aureus (MRSA) in an attempt to understand why there has been an epidemic spread of MRSA strains in Australian hospitals in recent years (Pavillard et al., 1982). Genetic analysis of these epidemic strains of MRSA revealed the presence of a particular class of plasmids encoding gentamicin resistance (Grubb et al., 1983; Townsend et al., 1983a, $1984 b$ and $1984 c$ ). The most common plasmid, of mol. wt $18 \times 10^{6}$, also codes for trimethoprim resistance. Initially, it was thought that this class of plasmid was primarily maintained by the MRSA for the gentamicin-resistance determinant. Subsequently, the transposable nature of this determinant was confirmed (Townsend et al., 1984c) and it now seems unlikely that gentamicin has been the selective agent for these plasmids (Grubb et al., 1983). Consequently, several antiseptics were investigated as possible selective agents.

This class of plasmid was found to encode resistance to cetyltrimethyl-ammonium bromide and propamidine isethionate (Townsend et al., 1984c). Although both these compounds are used in antiseptic preparations, it seemed unlikely that this is the reason for the spread of strains carrying such a resistance determinant. To characterise 
further the nature of resistance to these agents, various organic cationic compounds, including acriflavine and ethidium bromide, were examined. Resistance to acriflavine (Ericson, 1969) and ethidium bromide (Johnston and Dyke, 1969; Archer and Johnston, 1983; Foster, 1983; McDonnell et al., 1983) has been reported previously in $S$. aureus. The level of resistance to these compounds was compared in propamidinesensitive and -resistant strains that were isogenic except for the presence or absence of the propamidine-resistance determinant and other associated markers.

\section{MATERIALS AND METHODS}

Strains of $S$. aureus. The majority of strains of $S$. aureus studied have been described previously (Townsend et al., 1983b, 1984b and 1984c) and are detailed in table I. Strains WG1908, WG1762 and WG2936 are propamidine-resistant isolates of MRSA from Singapore, Ireland and Japan respectively. Their propamidine-resistance determinants have been transferred to recipient strains and their location analysed by previously published methods (Grubb et al., 1983; Townsend et al., $1983 b$ and 1984b). The transcipients generated were strains WG2306, WG2983 and WG3221 respectively (table I).

Determination of minimum inhibitory concentrations (MIC) of cationic agents. For MIC determinations, plates containing serial two-fold dilutions of each compound were prepared in

TABLE I

Plasmids and strains of Staphylococcus aureus

\begin{tabular}{|c|c|c|}
\hline Plasmid no. & Phenotype & Mol.wt $\left(10^{6}\right)$ \\
\hline $\begin{array}{l}\text { pWG115 } \\
\text { pWG53 } \\
\text { pWG54 } \\
\text { pWG401 } \\
\text { pWG202 }\end{array}$ & $\begin{array}{l}\mathrm{Pi}^{\mathrm{R}} \mathrm{Tp}^{\mathrm{R}} \\
\mathrm{Pi}^{\mathrm{R}} \mathrm{Gm}^{\mathrm{R}} \mathrm{Tp} \\
\mathrm{Pi}^{\mathrm{R}} \mathrm{Gm}^{\mathrm{R}} \\
\mathrm{Pi}^{\mathrm{R}} \mathrm{Nm}^{\mathrm{R}} \\
\mathrm{Pi}^{\mathrm{R}} \mathrm{Cd}^{\mathrm{R}} \mathrm{Hg}^{\mathrm{R}} \text { Pase }\end{array}$ & $\begin{array}{l}c .14 \cdot 6 \\
c .18 \cdot 0 \\
c .15 \cdot 3 \\
c .18 \cdot 0 \\
c .20 \cdot 0\end{array}$ \\
\hline Strain no. & Characteristics & Reference \\
\hline WG335 & Oxford strain, NCTC 6571 & \\
\hline RN450 & $\begin{array}{l}\text { Plasmid-free, non-lysogenic, } \\
\text { drug-sensitive }\end{array}$ & $\begin{array}{l}\text { Novick and } \\
\text { Bouanchaud, } 1971\end{array}$ \\
\hline WG541 & RN450 mutated to $\mathrm{Fa}^{\mathrm{R}} \mathrm{Rf}^{\mathrm{R}}$ & $\begin{array}{c}\text { Townsend et al, } \\
1983 b, 1984 c\end{array}$ \\
\hline WG822 & WG541, phage 95 lysogen & $\begin{array}{l}\text { Townsend et al, } \\
1983 b, 1984 b\end{array}$ \\
\hline $\begin{array}{l}\text { WG1876 } \\
\text { WG1437 }\end{array}$ & $\begin{array}{l}\text { WG541, phage J lysogen } \\
\text { MRSA isolated at Royal Perth } \\
\text { Hospital before } 1973\end{array}$ & $\begin{array}{l}\text { Townsend et al, } 1984 c \\
\text { Townsend et al, } 1984 a\end{array}$ \\
\hline WG588 & $\begin{array}{l}\text { MRSA isolated at Royal Prince } \\
\text { Alfred Hospital since } 1980 \\
\text { carrying pWG115 }\end{array}$ & Townsend et al, 1984c \\
\hline $\begin{array}{l}\text { WG2000 } \\
\text { WG935 }\end{array}$ & $\begin{array}{l}\text { WG1876+pWG } 115 \\
\text { WG588-pWGI15 }\end{array}$ & Townsend et al, 1984c \\
\hline WG525 & $\begin{array}{l}\text { MRSA isolated at Royal } \\
\text { Melbourne Hospital since } \\
1980 \text { carrying pWG53 }\end{array}$ & $\begin{array}{l}\text { Townsend et al, } \\
1983 b, 1984 c\end{array}$ \\
\hline $\begin{array}{l}\text { WG1320 } \\
\text { WG974 }\end{array}$ & $\begin{array}{l}\text { RN } 450+\text { pWG53 } \\
\text { WG } 822+\text { pWG53 }\end{array}$ & Townsend et al, 1984c \\
\hline WG686 & WG525-pWG53 & Townsend et al, $1983 \mathrm{~b}$ \\
\hline WG1451 & WG822+ pWG54 & Townsend et al, $1984 b$ \\
\hline WG2983 & WG822+pWG401 & $\cdots$ \\
\hline $\begin{array}{l}\text { WG2306 } \\
\text { WG3221 }\end{array}$ & WG1876+pWG202 & $\cdots$ \\
\hline 3221 & chromsomal P1" & $\cdots$ \\
\hline
\end{tabular}

$\mathrm{Pi}=$ propamidine isethionate, $\mathrm{Tp}=$ trimethoprim, $\mathrm{Gm}=$ gentamicin, $\mathrm{Nm}=$ neomycin, Pase $=$ penicillinase production, $\mathrm{Fa}=$ fusidic acid, $\mathrm{Rf}=$ rifampicin, superscript $\mathrm{R}=$ resistance. 
$15 \mathrm{ml}$ of Mueller-Hinton Agar (Gibco Diagnostics). Strains were grown overnight in Brain Heart Infusion Broth (Gibco), diluted 1 in 500 in $1 \%$ peptone water and then inoculated on to the test plates by the multipoint method. Plates were incubated overnight at $37^{\circ} \mathrm{C}$ and the lowest concentration of compound that prevented the appearance of visible growth was taken as the MIC.

For each strain of $S$. aureus, the MIC of the following compounds was determined: ethidium bromide, methylglyoxal bis(guanylhydrazone), spermine hydrochloride and spermidine tri-hydrochloride (Sigma); propamidine isethionate and amicarbalide isethionate (May and Baker); benzoflavin and coriphosphine $\mathrm{O}(\mathrm{K} \& \mathrm{~K}$ Laboratories Inc.); pyronin $\mathrm{Y}$, acridine yellow and crystal violet (Gurr, Searle Diagnostic); imidocarb dipropionate (Wellcome Australia Ltd); acriflavine (BDH Chemicals Ltd); cetyltrimethyl-ammonium bromide (Ajax Chemicals); acridine orange and safranin $\mathrm{O}$ (Chroma-Gesellschaft Schmid and Co.); chlorhexidine digluconate (Imperial Chemical Industries).

\section{RESULTS}

All strains of $S$. aureus carrying a resistance determinant to propamidine isethionate were also found to have increased resistance to several other cationic agents (table II). The level of resistance to these compounds was independent of other markers on the same plasmid. However, the MICs of these agents for strain WG3221, which carries the resistance determinant on the chromosome, were reduced by 2 - to 8 -fold in comparison with strains carrying plasmid-borne resistance.

The compounds exhibiting the greatest difference in MIC (up to a 128-fold increase) between resistant strains and their isogenic, sensitive derivatives were ethidium bromide, acriflavine, safranin $\mathrm{O}$, benzoflavin and propamidine isethionate (table II). Other compounds with an appreciable difference in MIC were pyronin Y, acridine yellow and imidocarb dipropionate. The resistance determinant conferred only a small increase in resistance to crystal violet (gentian violet), cetyltrimethylammonium bromide, amicarbalide isethionate, and methylglyoxal bis(guanylhydrazone). There was no significant difference between propamidine-resistant and -sensitive strains in their resistance to chlorhexidine digluconate, coriphosphine $O$, acridine orange, spermine or spermidine.

\section{Discussion}

Strains of $S$. aureus that were resistant to propamidine isethionate and cetyltrimethyl-ammonium bromide showed various degrees of cross-resistance to a broad range of cationic agents. An examination of the chemical structure of these compounds revealed several common features. Where the increase in MIC for a compound was more than 16-fold, the compound was symmetrical and contained two or more basic aromatic groups with terminal primary amine, tertiary amine, amidino or imidazole rings.

Despite the wide spectrum of compounds against which the resistance determinant was directed, relatively minor modifications in the chemical structure of the agents sometimes reduced significantly the degree of resistance. Thus, di-methyl substitution of one or both amine groups of acriflavine to form coriphosphine $\mathrm{O}$ or acridine orange respectively negated the effect of the resistance determinant. In fact, strains of $S$. aureus carrying the cationic-resistance determinant tended to be more sensitive than their isogenic derivatives to acridine orange.

Because of their basic nature, all the compounds whose resistance is coded for by 


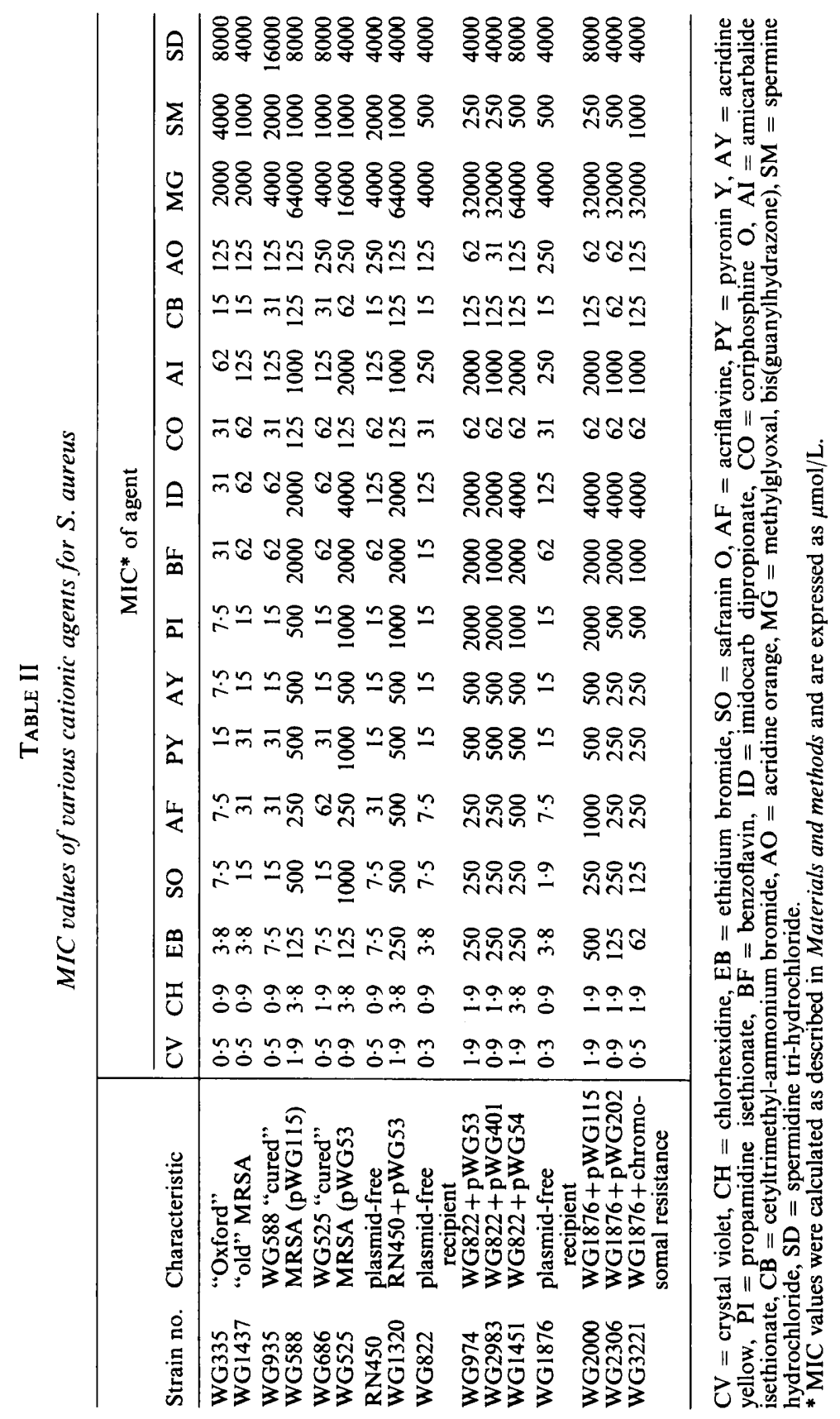


the determinant bind to a variety of anionic compounds including nucleic acids, mast-cell heparin and mucopolysaccharides. Bis(guanylhydrazones) (Denny and Cain, 1979), quaternary ammonium compounds (Scott, 1962) and diamidine compounds such as propamidine and amicarbalide (Anne et al., 1980) bind to nucleic acids, and the DNA-intercalating properties of ethidium bromide (Cain et al., 1978) and the acridines (Wolfe et al., 1972) have been well documented. This common property of binding to nucleic acids has prompted us to refer to the determinant encoding resistance to these compounds as the NAB (nucleic acid-binding compounds)-resistance determinant.

The antibacterial action of human semen has been attributed, at least in part, to the naturally occurring polyamines, spermine and spermidine (Razin and Rozansky, 1957) and the inhibitory action of these compounds on gram-positive cocci has been well documented (Rozansky et al., 1954; Fair and Wehner, 1971; Bachrach, 1973). The NAB-resistance determinant did not confer an increased resistance to spermine hydrochloride or spermidine tri-hydrochloride. In contrast, NAB-resistant strains of $S$. aureus tended to show an increased sensitivity towards spermine hydrochloride in comparison to their NAB-sensitive isogenic derivatives (table II).

Three representative isolates of MRSA were examined for their resistance to NAB agents. Strain WG1437, isolated from Royal Perth Hospital before 1973, is representative of the earlier strains of MRSA that lacked resistance to propamidine, whereas strains WG525 and WG588 were isolated after 1980 and carry a NAB-resistance determinant (Townsend et al., 1984b). The NAB-resistance profile of strain WG1437 was very similar to that of the Oxford strain, NCTC 6571, and other sensitive recipient strains such as RN450 and WG1876 (table II). Loss of the NAB-resistance plasmid from strains WG525 and WG588 (becoming strains WG686 and WG935 respectively) decreased their NAB resistance to that of sensitive organisms (table II).

Regardless of the source of the NAB-resistance determinant, the relative degree of resistance conferred to each compound studied was always similar. The decrease in levels of resistance displayed by strain WG3221, which carries chromosomal NAB-resistance, may reflect a gene dosage effect, because there may be more copies of the resistance determinant per cell when it is plasmid-borne than when it is located on the chromosome. The present results suggest that the NAB-resistance determinants present in the isolates of MRSA studied are similar and are distributed world-wide. They indicate that resistance to all the NAB compounds is conferred by the same determinant. Current studies will probably confirm this. Genetic determinants for resistance to acriflavine (Ericson, 1969) and ethidium bromide (Johnston and Dyke, 1969; Archer and Johnston, 1983; Foster, 1983; McDonnell et al., 1983) have been reported previously in $S$. aureus and they may be similar to the NAB-resistance determinant described here.

The use of agents such as acriflavine, propamidine, cetyltrimethyl-ammonium bromide and crystal violet in topical antiseptic creams may provide an explanation for the maintenance of the NAB-resistance determinant, but the current lack of widespread use of acriflavine and propamidine and the very small increase in resistance to cetyltrimethyl-ammonium bromide and crystal violet make this interpretation unlikely. We are now investigating several alternative possibilities that may indicate why this resistance determinant is carried by the MRSA strains currently being isolated in epidemic proportions in Australia (Pavillard et al., 1982). 
We thank May and Baker, Australia for donating the propamidine isethionate, Dr Tay Leng, Singapore, for strain WG1908, Professor E. C. Moorhouse, Dublin, Ireland for strain WG1762 and Dr M. Konno, Tokyo, Japan for strain WG2936. K.R.E. is the recipient of a National Health and Medical Research Council Australian Post-Doctoral Fellowship. This work was also funded in part by the Royal Perth Hospital Research Foundation, and by grants to W.B.G. from TVW Telethon and the National Health and Medical Research Council.

\section{REFERENCES}

Anne J, De Clercq E, Eyssen H, Dann O 1980 Antifungal and anti-bacterial activities of diarylamidine derivatives. Antimicrobial Agents and Chemotherapy 18:231-239.

Archer G L, Johnston J L 1983 Self-transmissible plasmids in staphylococci that encode resistance to aminoglycosides. Antimicrobial Agents and Chemotherapy 24:70-77.

Bachrach U 1973 Function of naturally occurring polyamines. Academic Press Inc., New York.

Cain B F, Baguley B C, Denny W A 1978 Potential antitumor agents. 28. Deoxyribonucleic acid polyintercalating agents. Journal of Medicinal Chemistry 21:658-668.

Denny W A, Cain B F 1979 Potential antitumor agents. 31. Quantitative structure-activity relationships for the antileukemic bis(guanylhydrazones). Journal of Medicinal Chemistry 22:1234-1238.

Ericson C 1969 Resistance to acriflavine and cadmium, and changed phage reactions: markers of a new staphylococcal penicillinase plasmid? Acta Pathologica et Microbiologica Scandinavica 76:333.

Fair W R, Wehner N 1971 Antibacterial action of spermine. Effect on urinary tract pathogens. Applied Microbiology 21:6-8.

Foster T J 1983 Plasmid-determined resistance to antimicrobial drugs and toxic metal ions in bacteria. Microbiological Reviews 47:361-409.

Grubb W B, Townsend D E, Greed L C, Ashdown N, Momoh M 1983 Characteristics of methicillin-resistant Staphylococcus aureus endemic in Australian hospitals. In: Spitzy K H, Karrer K (eds) Proceedings of the 13th International Congress of Chemotherapy, Verlag H. Egermann, Vienna. Part 83:23-26.

Johnston L H, Dyke K G H 1969 Ethidium bromide resistance, a new marker on the staphylococcal penicillinase plasmid. Journal of Bacteriology 100:1413-1414.

McDonnell R W, Sweeney H M, Cohen S 1983 Conjugational transfer of gentamicin-resistance plasmids intra- and interspecifically in Staphylococcus aureus and Staphylococcus epidermidis. Antimicrobial Agents and Chemotherapy 23:151-160.

Novick R P, Bouanchaud D 1971 Extrachromosomal nature of drug resistance in Staphylococcus aureus. Annals of the New York Academy of Science 182:279-294.

Pavillard R et al. 1982 Epidemic of hospital-acquired infection due to methicillin-resistant Staphylococcus aureus in major Victorian hospitals. The Medical Journal of Australia 1:451-454.

Razin S, Rozansky R 1957 The responsibility of spermine for the antibacterial action of human semen. Journal of Laboratory and Clinical Medicine 49:877-881.

Rozansky R, Bachrach U, Grossowicz N 1954 Studies on the antibacterial action of spermine. Journal of General Microbiology 10:11-16.

Scott J E 1962 The precipitation of polyanions by long-chain aliphatic ammonium salts. 6. The affinity of substituted ammonium cations for the anionic groups of some biological polymers. Biochemical Journal 84:270-275.

Townsend D E, Ashdown N, Annear D I, Pearman J W, Grubb W B $1984 a$ Genetic analysis of methicillin-resistant Staphylococcus aureus from a Western Australian hospital. Journal of Hospital Infection 5:417-424.

Townsend D E, Ashdown N, Greed L C, Grubb W B 1984b Analysis of plasmids mediating gentamicin resistance in methicillin-resistant Staphylococcus aureus. Journal of Antimicrobial Chemotherapy 13:347-352.

Townsend D E, Ashdown N, Greed L C, Grubb W B 1984c Transposition of gentamicin resistance to staphylococcal plasmids encoding resistance to cationic agents. Journal of Antimicrobial Chemotherapy 14:115-124. 
Townsend D E, Greed L C, Ashdown N, Grubb W B 1983a Plasmid-mediated resistance to quaternary ammonium compounds in methicillin-resistant Staphylococcus aureus. The Medical Journal of Australia 2:310.

Townsend D E, Grubb W B, Ashdown N $1983 b$ Gentamicin-resistance in methicillin-resistant Staphylococcus aureus. Pathology 15:169-174.

Wolfe A D, Allison R G, Hahn F E 1972 Labilizing action of intercalating drugs and dyes on bacterial ribosomes. Biochemistry 11:1569-1572. 